J Comp Physiol A (1986) 159:689-699

\title{
Control of echolocation pulses by neurons of the nucleus ambiguus in the rufous horseshoe bat, Rhinolophus rouxi
}

\author{
II. Afferent and efferent connections of the motor nucleus of the laryngeal nerves \\ Rudolf Rübsamen* and Hermann Schweizer \\ Zoologisches Institut der Universität München, Luisenstrasse 14, D-8000 München 2, Federal Republic of Germany
}

Accepted July 21, 1986

Summary. 1. Horseradish peroxidase was applied by iontophoretic injections to physiologically identified regions of the laryngeal motor nucleus, the nucleus ambiguus in the CF/FM bat Rhinolophus rouxi.

2. The connections of the nucleus ambiguus were analysed with regards to their possible functional significance in the vocal control system, in the respiration control system, and in mediating information from the central auditory system.

3 . The nucleus ambiguus is reciprocally interconnected with nuclei involved in the generation of the vocal motor pattern, i.e., the homonomous contralateral nucleus and the area of the lateral reticular formation. Similarly, reciprocal connections are found with the nuclei controlling the rhythm of respiration, i.e., medial parts of the medulla oblongata and the parabrachial nuclei.

4. Afferents to the nucleus ambiguus derive from nuclei of the "descending vocalization system' (periaqueductal gray and cuneiform nuclei) and from motor control centers (red nucleus and frontal cortex).

5. Afferents to the nucleus ambiguus, possibly mediating auditory influence to the motor control of vocalization, come from the superior colliculus and from the pontine nuclei. The efferents from the pontine nuclei are restricted to rostral parts of the nucleus ambiguus, which hosts the motoneurons of the cricothyroid muscle controlling the call frequency.

Abbreviations: $C F$ constant frequency; $D A B$ diaminobenzidine; $F M$ frequency modulation; $H R P$ horseradish peroxidase; $R L N$ recurrent laryngeal nerve; $S L N$ superior laryngeal nerve; $T M B$ tetramethylbenzidine

* Present address: Lehrstuhl für Allgemeine Zoologie, RuhrUniversität Bochum, Postfach 102148, D-4630 Bochum 1, FRG

\section{Introduction}

Microchiropteran bats produce their echolocation sounds in the larynx, which receives its motor innervation from two paired branches of the vagus nerve, the superior and the recurrent laryngeal nerves (SLN and RLN; Bowden and Scheuer 1961). In horseshoe bats it has been physiologically demonstrated that the frequency of the echolocation signals is controlled by the tension of a single laryngeal muscle, the cricothyroid, which is innervated by the motor branch of the SLN (Schuller and Suga 1976; Schuller and Rübsamen 1981). The recurrent laryngeal nerve (RLN) supplies the rest of the internal laryngeal muscles, especially the posterior cricoarytenoid and the lateral cricoarytenoid muscles which control the envelope of the sound, i.e., the start, duration, and the end of vocalization (Rübsamen and Schuller 1981). Horseshoe bats are an ideal model for the study of the neuronal control of vocalized sound parameters (see part I, Rübsamen and Betz 1986), as they emit stereotyped echolocation calls predominated by constant frequency components (CF, 30-90 ms in duration). On the one hand these CF components are emitted at individuum-specific frequencies ranging between 73 and $86 \mathrm{kHz}$, accurately maintained by each bat with a precision of $\pm 50 \mathrm{~Hz}$, on the other hand it is experimentally possible to cause the animals to vary the vocalized frequencies.

In mammals, motoneurons which innervate the muscles of the larynx via the laryngeal nerves are located in the nucleus ambiguus, the combined cranial nerve nucleus of the glossopharyngeal, the vagus, and the accessory nerves (Yoshida et al. 1982; Pasaro et al. 1983). In bats of the genus Rhinolophus the position of this nucleus in the caudal medulla oblongata has been shown by tracing the mo- 

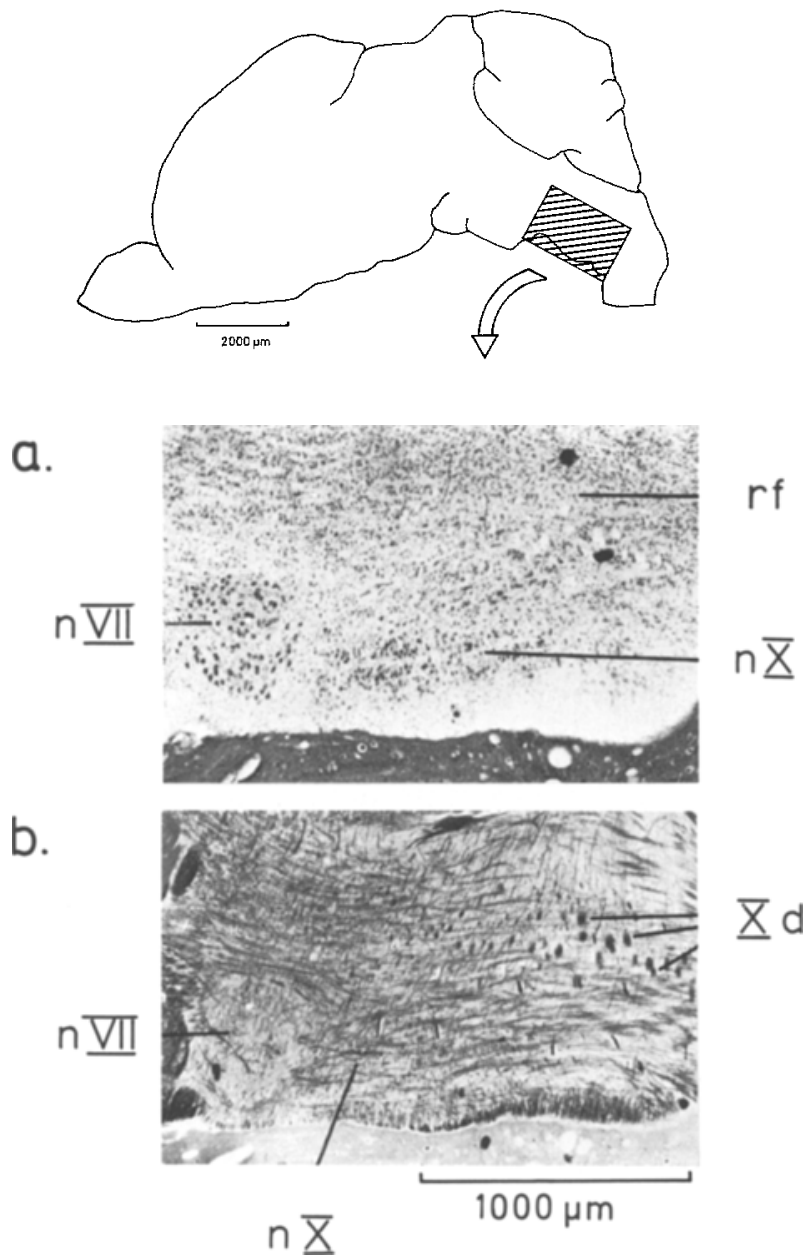

Fig. 1 a, b. Parasagittal section through the caudal medulla oblongata in the region of the nucleus ambiguus and the facial nerve nucleus (rostral to the left). a Cell-staining; b Fiber-staining. The nucleus ambiguus lies in direct caudal continuation of the facial nerve nucleus. $n V I I$ facial nerve nucleus; $n X$ nucleus ambiguus; $X d$ descending branches of the vagus; $r f$ reticular formation

toneurons with horseradish peroxidase (Schweizer et al. 1981). The nucleus ambiguus is located in direct caudal continuation of the facial nerve nucleus embedded in the reticular formation, from which it can not be strictly demarcated in stained brain slices (Fig. 1). However, HRP-studies have shown that the motoneurons of the SLN and RLN are clearly separated within the nucleus. Motoneurons which innervate the cricothyroid muscle via the superior laryngeal nerve and control the frequency of the echolocation calls, are found in the rostral part of the nucleus, and those which supply all the other internal laryngeal muscles via the RLN and control the pulse envelope, are located in its caudal part. Both groups of motoneurons overlap in wedge-shaped formations in the middle parts of the nucleus. The axons of all motoneurons directed to the larynx leave the medulla oblongata without forming collaterals (Barillot et al. 1984).

Considering the neuroanatomical results from different mammals, the afferent and efferent connections of the nucleus ambiguus can be summarized as follow. The laryngeal motor nucleus receives inputs from the periaqueductal gray (Jürgens and Pratt 1979), and from parts of the nucleus of the solitary tract (Kalia 1981; Sawchenko 1983). Reciprocal interconnection has been demonstrated between the nucleus ambiguus and the parabrachial nuclei (Saper and Loewy 1980; Kalia 1981). The above mentioned periaqueductal gray is known to be part of the "descending vocalization pathway' (Jürgens and Pratt 1979), whereas the parabrachial nuclei and parts of the nucleus of the solitary tract are involved in the generation of respiratory rhythm (von Euler 1983).

In horseshoe bats, for which a direct control of vocalized frequencies depending on the auditory input signal has been demonstrated (Schuller 1977), the knowledge of the interconnection between the auditory and the vocalization system is of particular interest. To gain information about the central linkage between the two subsystems involved in echolocation, and to understand the central organization of the vocal motor control system in bats, we applied HRP tracing methods to physiologically identified neuron populations of the nucleus ambiguus and analysed the afferent and efferent connections of this nucleus.

\section{Materials and methods}

Of the sixteen rufous horseshoe bats (Rhinolophus rouxi) which underwent the neurophysiological experiments (described in Part I., Rübsamen and Betz 1986) six were used to trace the anatomical connections of the nucleus ambiguus.

After physiologically identifying the neurons in different areas of the laryngeal motor nucleus, HRP (Sigma Type VI) was iontophoretically injected. Glass micropipettes bevelled to a tip diameter of 5-15 $\mu \mathrm{m}$ and filled with $10 \%$ or $20 \%$ HRP dissolved in distilled water were used for iontophoresis. To produce small injection sites (about 200 to $400 \mu \mathrm{m}$ in diameter) a positive current of 800 to $900 \mathrm{nA}$ was applied to the electrode for 3 to $4 \mathrm{~min}$. In some experiments the injection time was prolonged for up to 8 to $12 \mathrm{~min}$ which led to large injection spots of about $500 \mu \mathrm{m}$ in diameter.

After 20 to $24 \mathrm{~h}$, the animals were fatally anaesthetized by an overdose of Nembutal ( $5 \mathrm{mg} / 100 \mathrm{~g}$ body weight), and fixed by transcardial perfusion (at the rate of $4 \mathrm{ml} / \mathrm{min}$ ) of $1.25 \%$ glutaraldehyde and $1 \%$ paraformaldehyde in $0.1 \mathrm{M}$ phosphate buffer, pH 7.2 (Rosene and Mesulam 1978). A small amount of $2.5 \%$ sucrose was added to the fixative. After $50 \mathrm{~min}$ the fixative was replaced by $0.1 M$ phosphate buffer ( $\mathrm{pH}$ 7.2), with increasing concentrations of sucrose $(5 \%, 10 \%, 20 \%)$.

After perfusion, the brain and upper parts of the cervical spinal cord were dissected out and embedded in egg yolk, mixed 
with glutaraldehyde $(0.7 \mathrm{ml}$ [25\%] glutaraldehyde $/ 10 \mathrm{ml}$ egg yolk) on a magnetic stirrer $(5-7 \mathrm{~s})$. This procedure was performed in a small rectangular embedding chamber that allowed the introduction of markers, and later on, an appropriate orientation of brain sections for serial reconstruction.

The brains were immediatelly frozen with dry ice and sections, $48 \mu \mathrm{m}$ thick, were cut in transverse or parasagittal planes in a cryostat. Three series of sections were collected in $0.1 \mathrm{M}$ phosphate buffer ( $\mathrm{pH} 7.2$ ), one of which was treated the same day with tetramethylbenzidine (TMB; Mesulam and Rosene 1979). The sections were mounted on gelatinized slides, dehydrated and covered with cover-slips without counterstaining. The second series was treated with diaminobenzidine (DAB; Adams 1977), and later counterstained with neutral red. The third series was directly mounted on slides and stained with cresylviolet, to serve as reference for reconstruction and identification of the brain structures.

Every second section of the cresylviolet-stained reference series was viewed through a microscope equipped with a drawing tube and traced on paper. HRP-labelled structures (fibers and neurons) of the neighbouring sections (from the two histochemically treated series) were then graphically superimposed. For distance measurements in the transverse series the caudal end of the facial nerve nucleus served as reference, and in the parasagittal series the midsagittal plane was the reference.

\section{Results}

HRP injections were made into different parts of the nucleus ambiguus in six horseshoe bats. The neurotracer was located either in the intermediate part of the nucleus ambiguus, hosting the RLN as well as the SLN motoneurons, or in the rostral part of the nucleus containing the SLN motoneurons and interneurons, both of which had activities gradually changing with the echolocation pulse frequency (see Part I., Rübsamen and Betz 1986). None of the injections were exclusively restricted to the caudal RLN area. In all experiments the injections hit the ventral motor nucleus of the vagus, and were restricted only to this nucleus, as was seen from the DAB reacted sections. But, estimating the HRP deposit spots from sections treated with the more sensitive TMB method, it became apparent that the injection sites also included parts of the neighbouring reticular formation.

Nevertheless, the size of the HRP-injections in different experiments were estimated from DABreacted material. This, in contrast to the TMBmaterial, allowed a clear-cut delimitation of the injection sites, which served as an aid for the comparison of results obtained from different experiments. DAB-reacted sections also allowed the differentiation between anterograde and retrograde neuronal transport of the enzyme within the region of the medulla oblongata and up to the midbrain. The Golgi-like staining clearly displayed cell somata showing the retrograde transport of HRP, and labelled fibers fanning out in terminal fields demonstrating anterograde transport. Golgi-like labelling also enabled the tracing of the course of fibers (anterograde and retrograde), interconnecting the injected nucleus ambiguus with other nuclei. The transport of the enzyme to the diencephalon and to more rostral areas, as well as to posterior parts of the cervical spinal cord were analysed from TMB-treated material. This enabled a reliable identification of retrogradely labelled cell somata afferent to the injected nucleus ambiguus, whereas labelled fibers and terminal fields showed diffuse occurrence and were, despite one unmistakable example in the cervical spinal cord, not taken into account.

Injections into the intermediate region of the nucleus ambiguus ( $R L N$ and SLN motoneurons)

In four experiments (NA10, NA11, NA12 and NA13) the HRP-injections were located in the middle part of the nucleus ambiguus. The centers of the injections were located within an area of $450 \mu \mathrm{m}$ to $1,000 \mu \mathrm{m}$ posterior to the caudal end of the facial nerve nucleus (Figs. 2f, 3e). The diameter of the injection spots varied between 350 and $400 \mu \mathrm{m}$.

\section{Afferent projections}

In all four experiments the pattern of retrogradely labelled neurons (i.e. afferents to the nucleus ambiguus) was found to be very similar (Figs. 2, 3).

Medulla oblongata. The most prominent retrograde labelling in the brain stem was found ipsilateral to the injection site in the parabrachial nucleus, where small labelled cells were densely packed around the superior cerebellar peduncle (Figs. 2e, $3 \mathrm{f})$. In the joined region of the lateral reticular formation, between the parabrachial nucleus and the motor nucleus of the larynx, retrogradely labelled cells were scattered between the area of the facial nerve nucleus and the motor nucleus of the trigeminal nerve. On the contralateral side the same pattern of labelling was observed, but somewhat reduced (Figs. 2e, 3b). Ipsilateral to the injection site, labelled cells were found in the nucleus ambiguus over its total rostrocaudal extension including the retroambigual area which forms the link to the ventral horn of the cervical spinal cord (Fig. 3e). Some labelled cells were also found scattered in this area. Many labelled neurons were located in the contralateral nucleus ambiguus from its rostral to its caudal pole (Fig. $3 \mathrm{~b}$ ). In addition, 
a]

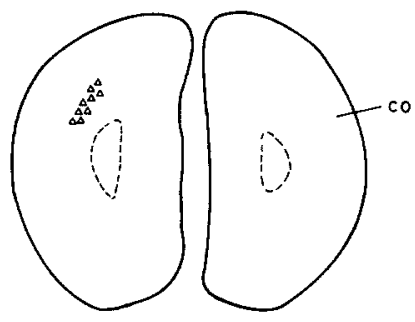

b

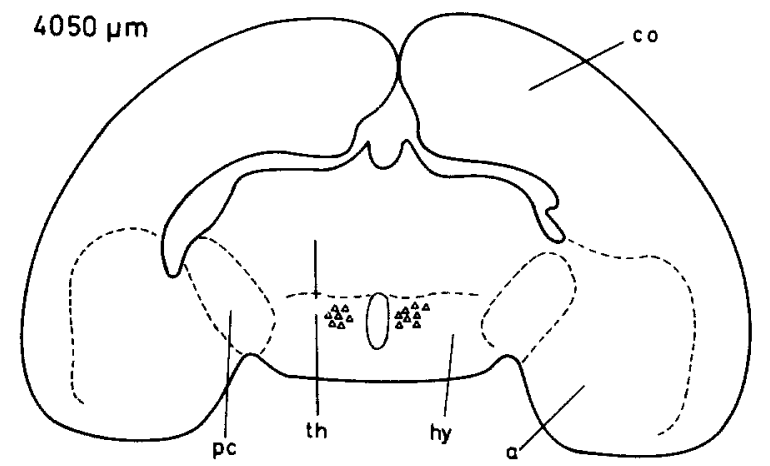

e
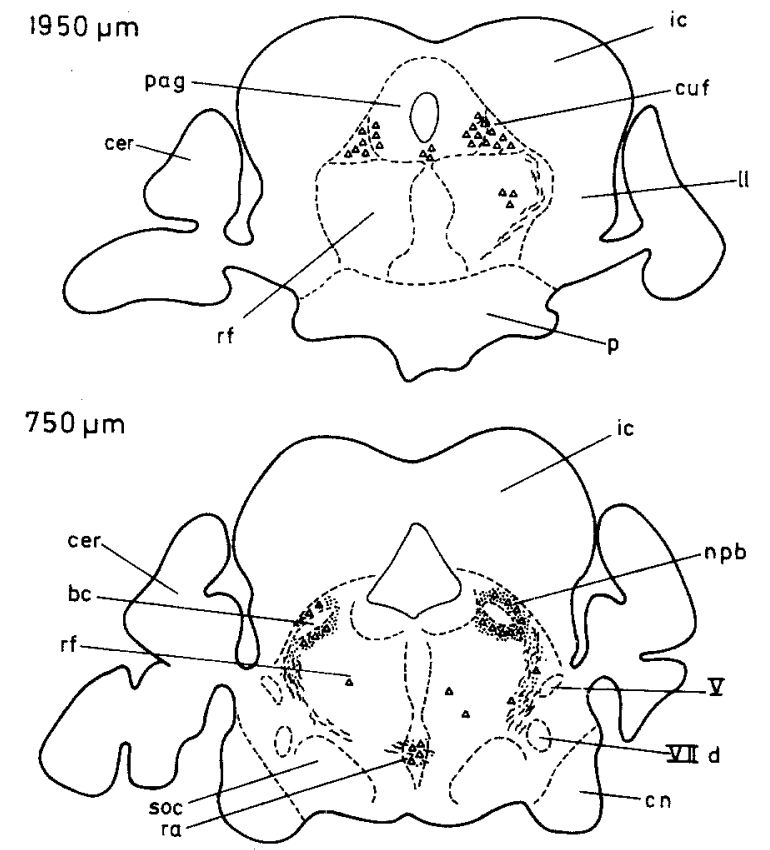

$50 \mu \mathrm{m}$
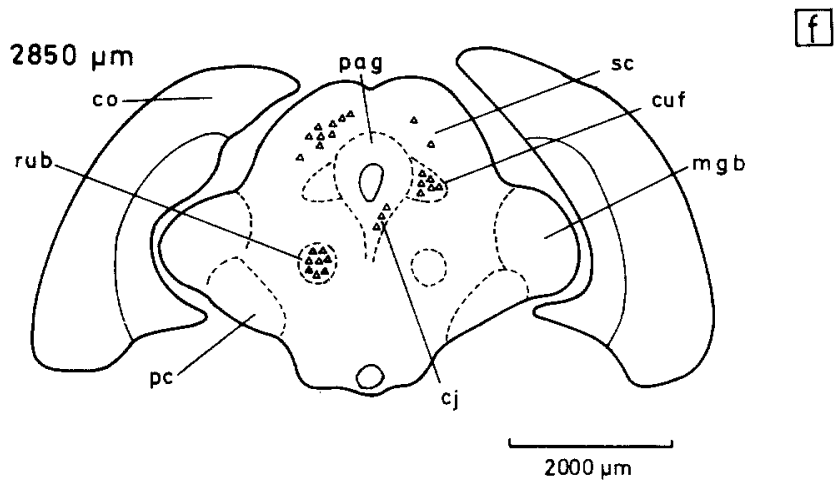

$-750 \mu \mathrm{m}$

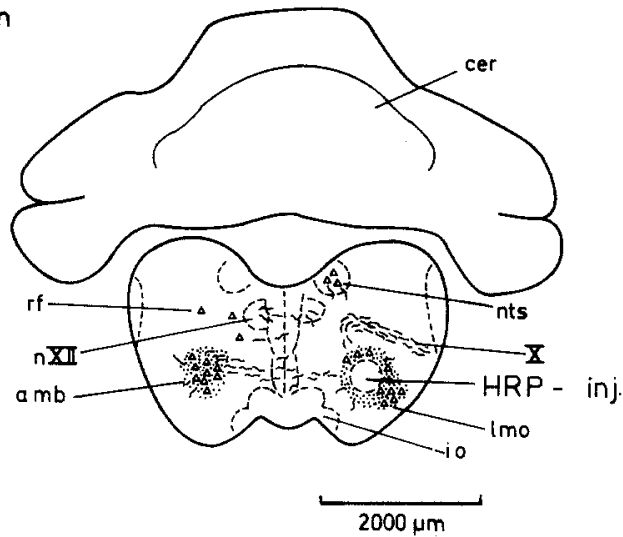

Fig. 2 a-f. Schematic drawings of transverse sections demonstrating the anterograde and retrograde labelling by the reaction product following HRP injection into the nucleus ambiguus $750 \mu \mathrm{m}$ (Na12) caudal to the facial nerve nucleus (HRP-inj. at $-750 \mu \mathrm{m})$. Retrograde labelling is shown by triangles, labelled fibers by interrupted lines. Terminal fields, which had been marked by anterograde transport, are demonstrated by dotted areas. The order of the sections is in relation to the caudal end of the facial nerve nucleus (distance in $\mu \mathrm{m} ;[+]$ to the rostral side, $[-]$ to the caudal side). Explanation of abbreviations: $a$ amygdala; $a m b$ nucleus ambiguus; $b c$ brachium conjunctivum; $c e r$ cerebellum; $c j$ interstitial nucleus of Cajal; $c n$ cochlear nucleus; co cerebral cortex; cuf cuneiform nucleus; hy hypothalamus; ic inferior colliculus; io inferior olive; $l l$ lateral lemniscus; lmo lateral nucleus of the medulla oblongata; $m g b$ medial geniculate body; $n p b$ parabrachial nucleus; $n t s$ nucleus of the solitary tract; $n X I I$ hypoglossal nerve nucleus; $p$ pons; pag periaqueductal gray (central gray matter); $p c$ cerebral peduncle; $r a$ raphe magnus; $r f$ reticular formation; $r u b$ red nucleus; $s c$ superior colliculus; soc superior olive; th thalamus; $V$ efferent fibers of the trigeminal nerve; VIId descending tract of the facial nerve; $X$ efferent vagal fibers

lightly labelled cells were detected in the ipsilateral nucleus of the solitary tract, dorsal to the nucleus ambiguus, in the lateral nucleus of the medulla oblongata ventral to the injected nucleus, and in the raphe nuclei at a midline position (Fig. $2 \mathrm{e}, \mathrm{f}$ ).

Mesencephalon. In the midbrain region, numerous neurons were labelled bilaterally in the cuneiform nucleus, in the lateral area of the periaqueductal gray, and scattered within the deep layers of the superior colliculus (Fig. 2c, d), whereas contralaterally labelled cells were found in the red nucleus. The labelled neurons in the lateral periaqueductal gray form bilaterally arranged rostrocaudal cell bands, about $750 \mu \mathrm{m}$ in length, located just medially to the labelled neurons in each of the cuneiform nuclei. The interstitial nucleus of Cajal and the mesencephalic nucleus of the trigeminal nerve, 

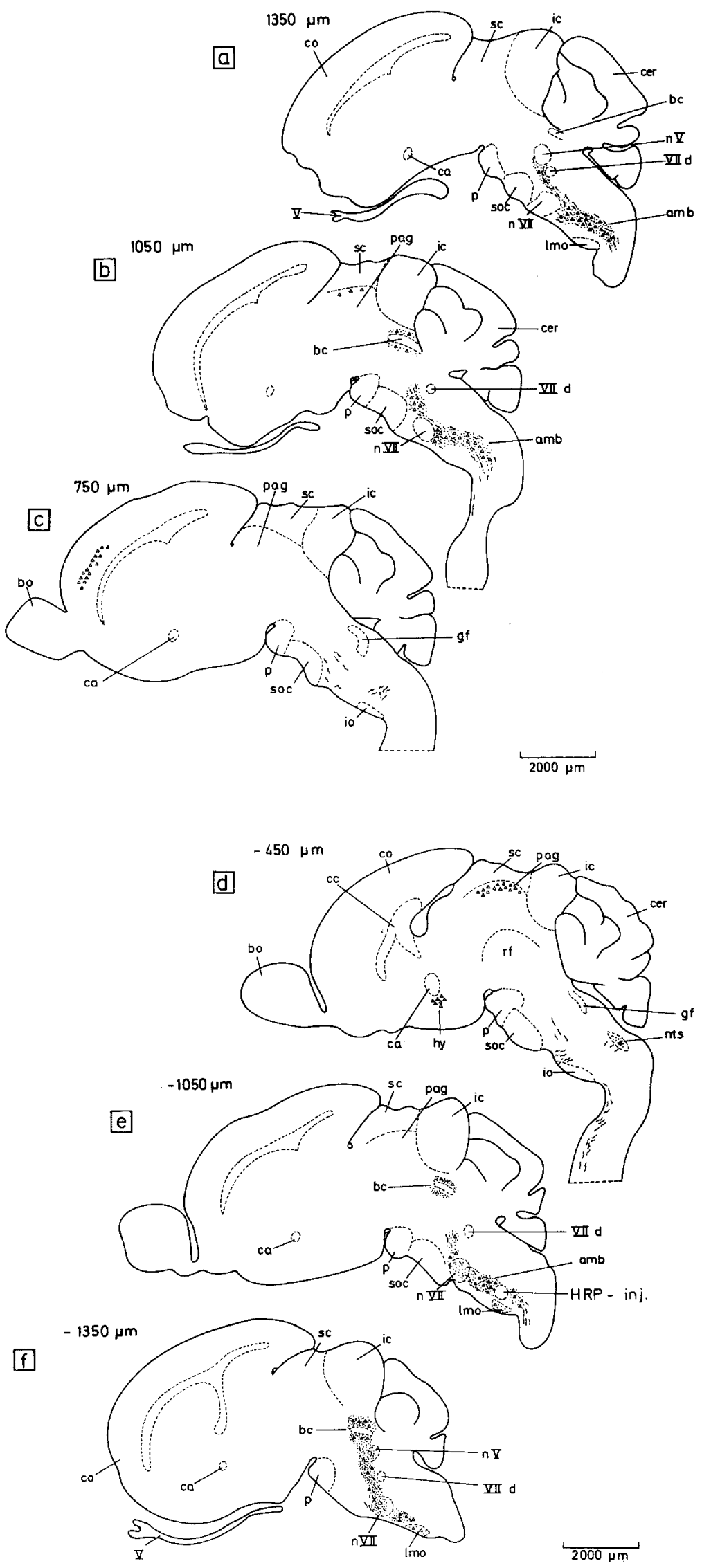

Fig. 3a-f. Schematic drawings of parasagittal sections demonstrating anterograde and retrograde labelling by the reaction product following HRP injection into the intermediate region of the nucleus ambiguus (Na10), (HRP-inj. at $-1,050 \mu \mathrm{m})$. Retrograde labelling shown by triangles, labelled fibers by interrupted lines. Terminal fields, marked by anterograde transport, are demonstrated by dotted areas. The series of sections are related to the midline of the caudal brain stem (distance in $\mu \mathrm{m} ;[-]$ ipsilateral to the injection site, $[+]$ contralateral to the injection site). Explanation of abbreviations: $a m b$ nucleus ambiguus; $b c$ brachium conjunctivum; $b o$ olfactory bulb; $c a$ anterior commissure; $c c$ corpus callosum; cer cerebellum; co cerebral cortex; gf genu facialis; hy hypothalamus; ic inferior colliculus; io inferior olive; lmo lateral nucleus of the medulla oblongata; nts nucleus of the solitary tract; $n V$ motor nucleus of the trigeminal nerve; $n V I I$ facial nerve nucleus; $p$ pontine nuclei; pag periaqueductal gray (central gray matter); $s c$ superior colliculus; soc superior olive; $V$ trigeminal nerve; VIId descending tract of the facial nerve 
ipsilateral to the injection site, also contained neurons filled with the reaction product (Figs. $2 \mathrm{c}, 3 \mathrm{f}$ ).

Diencephalon. In the ventral hypothalamus two groups of neurons contained the reaction product, forming wedge shaped areas on both sides of the third ventricle (Figs. 2b, 3d).

Telencephalon. Labelled pyramidal cells were found in layer $\mathrm{V}$ at the rostral-most pole of the cerebral cortex, contralateral to the injection side (Figs. 2a, 3c).

This projection pattern was identical in all experiments of this group, with the exception of experiment NA11, where labelled neurons were also bilaterally found in the lateral pontine nuclei. In this experiment the injection site was situated more rostrally in the nucleus ambiguus, $450 \mu \mathrm{m}$ from the caudal pole of the facial nerve nucleus, and thus included parts of the rostral SLN-area.

\section{Efferent projections}

In all experiments, fibers which were labelled took their origin at the injection site and travelled in different directions, sometimes combined in compact fiber bundles. Part of these fibers possibly were axons of retrogradely labelled neurons, but unequivocally many were anterogradely labelled axons of neurons located in the nucleus ambiguus, or in the direct adjacent reticular formation. This was elucidated by the Golgi-like staining of multiple fiber bifurcations and of the terminal fields in DAB-treated sections. The visualization of the efferent projection pattern may be incomplete as it is known that anterograde transport, as shown by the DAB method, is only demonstrable over a restricted distance.

The labelled fibers travelled mainly in four directions:

(1) The majority of efferent fibers originating from the injection spot ascended rostrally on the ipsilateral side, running around the facial nerve nucleus. On their course to the parabrachial nucleus, the fibers accumulated in a dense bundle, which ran below the descending branch of the facial nerve and continued in a position lateral to the motor nucleus of the trigeminal nerve. On their way through the lateral reticular formation (ipsilateral to the injection site) many collaterals bifurcate from the main course, forming fiber terminals and ending here (Figs. 2e, 3f). This region was identical to that part of the reticular formation where retrogradely labelled cell somata had been found after HRP-injection in the nucleus ambiguus. Thus it represents an area with strong afferent and efferent connections to the nucleus ambiguus.

(2) The second fiber bundle, composed of efferents from the nucleus ambiguus, ran caudally through the nucleus itself. Many fibers terminated within the nucleus ambiguus (Fig. $3 \mathrm{~b}$ ), while others continued caudally through the nucleus and joined the lateral funiculus of the cervical spinal cord, ipsilateral to the injection spot.

(3) The third bulk of efferent fibers crossed the midline towards the contralateral nucleus ambiguus (Fig. 2f). These formed terminals along the entire rostrocaudal extension of this nucleus, from the facial nerve nucleus up to the retroambigual nucleus and to the ventral funiculus of the cervical spinal cord (Fig. 3a). Some labelled fibers ran through the contralateral nucleus ambiguus and continued dorsorostrally in the lateral reticular formation, showing generally the same fiber arrangement, but less densely labelled when compared with that in the lateral reticular formation ipsilateral to the injection site (Fig. $3 \mathrm{~b}$ ).

(4) Starting from the injection spot, labelled axons of laryngeal motoneurons ascended ipsilaterally in a dorsomedial direction towards the dorsal motor nucleus of the vagus nerve. Near the border of this nucleus the fibers bent ventrolaterally and left the medulla oblongata in a lateral position integrated in bundles of the vagus nerve (Fig. 2f).

In addition to these four closely packed fiber aggregations, many single fibers leaving the injection spot were found to terminate ipsilaterally in the neighbouring reticular formation, in the lateral nucleus of the medulla oblongata, in the midline raphe, in the facial nerve nucleus and in the motor nucleus of the trigeminal nerve. Some fibers terminated bilaterally in the hypoglossal nerve nucleus.

Injections into the rostral region of the nucleus ambiguus (SLN motoneurons)

In two experiments (NA9 and NA15) the injections were made in the rostral area of the nucleus ambiguus, about $250 \mu \mathrm{m}$ from the caudal pole of the facial nerve nucleus. Both injection spots were slightly larger than in other experiments (about $500 \mu \mathrm{m}$ in diameter), and included parts of the surrounding reticular formation. They may also have affected fibers which travel near the nucleus ambiguus, many of which are pyramidal tract fibers.

Anterograde (efferent) and retrograde (afferent) projection patterns obtained in these experiments were similar to those described above. The 
main difference found in both preparations was additional retrograde labelling of neurons bilaterally located in the lateral pontine nuclei (Fig. 4).

In both experiments retrograde labelling was more intense in the interstitial nucleus of Cajal and the superior colliculus when compared with injections in the middle area of the nucleus ambiguus.

In these two experiments it was also possible to reconstruct the complete course of the pyramidal system, by which forebrain inputs might be mediated to the nucleus ambiguus. The region of labelled pyramidal neurons was situated in layer $\mathrm{V}$ of the frontal cortex, starting at its rostral-most pole and extending $2,500 \mu \mathrm{m}$ rostrocaudally. The fibers descended in the cerebral peduncle and travelled through the pons. The pyramidal decussation was located at the level of the facial nerve nucleus. In between this nucleus and the nucleus ambiguus, pyramidal fibers crossed to the contralateral side. They ran in a dispersed formation through the medulla oblongata, directly lateral to the nucleus ambiguus, branching to short collaterals that form fiber terminals. The pyramidal tract aggregated again when it entered the cervical spinal cord.

In TMB-treated sections, terminal granulations, demonstrating anterograde transport of the enzyme were seen in the ventral horn, up to a region $5,000 \mu \mathrm{m}$ caudal to the inferior olive. No labelled fibers or terminals were further caudally detected. In rhinolophids the pyramidal tract running in the funiculus lateralis ends in the cervical spinal cord.

\section{Scheme of the connections of the nucleus ambiguus and the adjacent reticular formation}

Figure 5 summarizes the afferent and efferent connections of the nucleus ambiguus as derived from the experiments described above. Here they are described in their arrangement from rostral to caudal positions: the frontal cortex pole projects contralaterally to the nucleus ambiguus. Bilateral afferents to the nucleus ambiguus come from the ventral hypothalamus. Afferents from the red nucleus originate on the contralateral side and those from the interstitial nucleus of Cajal and from the mesencephalic nucleus of the trigeminal nerve on the ipsilateral side. The afferents from other midbrain structures are always bilaterally organized, but emphasized in the ipsilateral side. These nuclei are the periaqueductal gray, the cuneiform nuclei and the superior colliculi. Afferents from the lateral pontine nuclei are bilaterally organized. The parabrachial nuclei show afferent as well as efferent connections with the motor nucleus of the larynx.

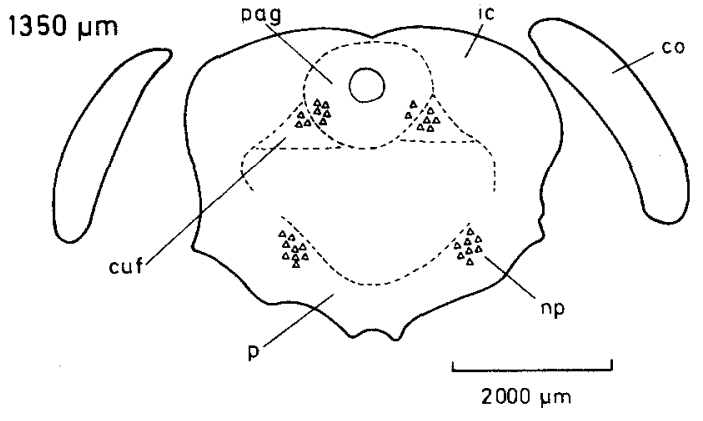

Fig. 4. Schematic drawing of a transverse section in the midbrain region $(1,350 \mu \mathrm{m}$ rostral to caudal end of the facial nerve nucleus) demonstrating retrograde labelling by the reaction product following HRP injection into the frontal area of the nucleus ambiguus. Retrograde labelling shown by triangles. Explanation of abbreviations: cuf cuneiform nucleus; co cortex; ic inferior colliculus; $n p$ pontine nuclei; $p$ pons; pag periaqueductal gray

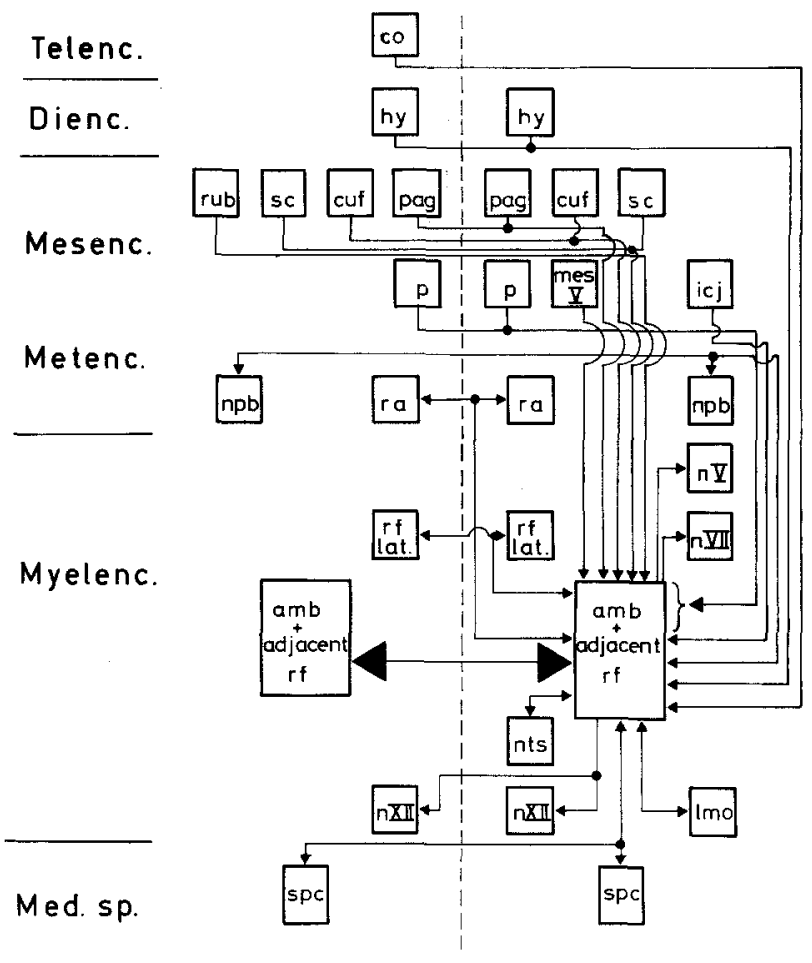

Fig. 5. Schematic representation of the afferent and efferent connections of the nucleus ambiguus and the adjacent reticular formation. For further explanations see text. For explanations of the abbreviations of neuroanatomical terms see Fig. 2

The same holds for the midline raphe and the lateral system of the reticular formation. The motor nucleus of the trigeminal nerve and the facial nerve nucleus receive ipsilateral input from the nucleus ambiguus. Very strong reciprocal connections are seen between the homonomous motor nuclei of the larynx. Weaker afferent connections with the nucleus ambiguus, arranged ipsilaterally, are dem- 
onstrated for the nucleus of the solitary tract and in the lateral nucleus of the medulla oblongata, whereas the cervical spinal cord has bilateral reciprocal connections. Efferents from the motor nucleus of the larynx reach the nuclei of the hypoglossal nerve on both sides.

\section{Discussion}

In the experiments reported here, we have identified those brain structures which connect to or receive fibers from the nucleus ambiguus. The physiological data suggest that the nucleus ambiguus is composed of laryngeal motoneurons and also of neurons which project to other brain structures (Rübsamen and Betz 1986). However, this nucleus cannot be strictly demarcated from the surrounding reticular formation and many fibers on their course through the medulla oblongata travel through the nucleus ambiguus itself (Fig. 1). Therefore some brain structures may be labelled by tracer uptake from passing fibers, and/or by tracer diffusion to the reticular formation, thereby hampering a clear-cut interpretation of the data presented. In spite of this uncertainty, the data show that the laryngeal motor center is connected with four functional systems: (1) the descending vocalization pathway, (2) the auditory system, (3) respiration centers, and (4) the reticular system.

\section{Vocalization pathways}

In all injections that pervaded the area of the laryngeal motoneurons, strong retrograde and anterograde labelling was found in the lateral reticular formation of the medulla oblongata up to the midbrain level. The area rostrodorsal to the nucleus ambiguus, including the lateral reticular formation around the facial nerve nucleus, the retrofacial nucleus, and the parabrachial nucleus, contains labelled neurons and axon terminals. Therefore these medullary nuclei seem to be intricately interconnected with the nucleus ambiguus: the laryngeal motor nucleus receives inputs from these areas and projects back to them. In squirrel monkeys artificial vocalizations, never spontaneously emitted by the animals, could be evoked in the lateral reticular formation by electrical stimulation (Jürgens and Pratt 1979). This part of the reticular formation seems to play an important role in the generation of motor patterns for vocalization. Furthermore, it is evident in squirrel monkeys, that all motor nuclei which are involved in the motor control of articulation (i.e. the facial nerve nucleus, the trige- minal motor nucleus, the motor nucleus of the hypoglossal nerve and the nucleus ambiguus), receive inputs from this region of the reticular formation (Jürgens, personal communication).

Another brain center which is known to be involved in the generation of vocalization, is the periaqueductal gray of the midbrain (Jürgens and Ploog 1970; Suga et al. 1973). Neurons projecting from this midbrain nucleus to the nucleus ambiguus are bilaterally located in small cellular bands restricted to its lateral parts, in direct continuation with labelled cells in the neighbouring cuneiform nuclei. In bats, vocalizations were elicited by electrical stimulation of these structures, with the shortest latencies (20 to $60 \mathrm{~ms}$ ) and the lowest effective current reported for the vocalization system (Suga et al. 1973; Schuller 1979). Also in squirrel monkeys, the periaqueductal gray has a prominent role in the vocalization system. This nucleus is not directly incorporated in the somatomotor or visceromotor fiber tracts of the extrapyramidal or pyramidal motor systems. But it is connected with all prosencephalic vocalization centers, and lesions in this nucleus mute the animals (Jürgens and Pratt 1979). The periaqueductal gray is an obligatory relay station for the control of vocalization. By injection of an anterograde neurotracer it could be demonstrated that efferents arising here reach the complete lateral reticular formation of the medulla oblongata, including the nucleus ambiguus and the region in which this nucleus is embedded (Jürgens and Pratt 1979). As illustrated in our experiments in bats, this region of the lateral reticular formation is, in addition, reciprocally interconnected with the nucleus ambiguus.

Many neuroanatomical studies using tracing techniques have been directed at the nociceptive system, in which the periaqueductal gray also plays an important role. In such investigations, afferent and efferent connections of this area with the lateral reticular formation of the medulla oblongata (similar to those found by us) have been reported (Chung et al. 1983; Manthy 1982, 1983). Data for the connections of the cuneiform nucleus (considered to be the reticular formation of the midbrain) with the caudal medulla oblongata are also congruent with our results (Edwards 1975; Abols and Basbaum 1981).

The incorporation of the periaqueductal gray and of the nucleus cuneiformis in the "descending vocalization pathway' demands further clarification. A single unit analysis in vocalizing bats with an exact localization of the recording sites would enable a direct comparison with laryngeal premotor and motor activity reported for rhinolophids 
(Rübsamen and Betz 1986), and would help to understand the generation of the motor control pattern for vocalization.

The retrograde labelling of the contralateral red nucleus and the contralateral cortex shows the connection of the "vocalization pathway' with the extrapyramidal and pyramidal motor system. But it can not be excluded that also via the red nucleus an auditory influence, not mediated by the forebrain, reaches the motor control system for vocalization. At least in decerebrated cats auditory evoked activities could be recorded in the red nucleus (Irvine 1980).

Labelled neurons in the contralateral telencephalon were restricted to the rostral cortex pole. This region belongs to the motor cortex, as might be concluded from a comparative neuromorphological analysis of the neocortex in bats and in insectivores (Rose 1912), and from neurophysiological investigations of the forebrain in insectivores (Lende 1969). This suggests that the nucleus ambiguus receives direct input from the pyramidal motor system, a possibility that does not correspond with data so far described in the literature. Among mammals, only in primates the visceral nerve nuclei allegedly receive direct input from the pyramidal tract (Kuypers 1958, 1960). From studies in cats and rats (Walberg 1957; Valdere 1962) it was concluded that, in all mammals of lower phylogenetic rank, the corticofugal fibers to the caudal medulla oblongata should be mediated to the visceral nerve nuclei via the reticular formation.

Although we cannot exclude with absolute certainty that the pyramidal fibers terminate within the surrounding reticular formation, in bats a direct input to the nucleus ambiguus seems possible. For Microchiroptera Henson (1970) has already suggested a direct connection of the pyramidal tract to the nucleus ambiguus. His arguments were the reduction of number of pyramidal fibers in the transition region of the medulla oblongata to the lateral funiculus. Our findings support this suggestion and show that no pyramidal fibers extend beyond the cervical spinal cord. Further support for a direct pyramidal input to the ventral motor nucleus of the vagus comes from the fact that in rhinolophids the pyramidal decussation is located at the level of the facial nerve nucleus, thus far more rostrally in comparison with other mammals. Thus the pyramidal fibers run along the side of the nucleus ambiguus over its whole rostrocaudal extent, and moreover, these fibers show a dispersed organization in that region. Collaterals branch from these fibers and form terminals in the region of the nucleus ambiguus and the neighbouring reticular formation.

\section{Auditory connections}

Single unit recordings in the nucleus ambiguus have given evidence for auditory input to the motor nucleus of the larynx (Rübsamen and Betz 1986). Midbrain nuclei that may mediate auditory afferents are the superior colliculus and the lateral nuclei of the pons. Labelled cells were found in both nuclei after HRP-injections into the nucleus ambiguus, and in the Greater Horseshoe bat it has been shown that both receive direct inputs from the inferior colliculus (Schweizer 1981).

Cells scattered in deep layers of the superior colliculus project directly to the nucleus ambiguus and the adjacent reticular formation. In the superior colliculus neurons processing directional auditiory information have been reported in bats (Shimozawa et al. 1984), as well as in cats (Middelbrooks and Knudsen 1984). A connection from this nucleus to the paralemniscal zone (lateral reticular formation) has been demonstrated in cats. In the same species the colliculus superior itself projects to the facial nerve nucleus mediating auditory information for the control of pinna movements (Henkel and Edwards 1978; Henkel 1981).

Hindbrain regions that possibly mediate auditory input to the vocalization system are the lateral nuclei of the pons. Here Boyd and Aitkin (1976) recorded single unit activities correlated with auditory stimuli with short latencies, comparable to acoustically activated single units of the cerebellum (Aitkin and Boyd 1975). In our experiments in rhinolophids we found that anterior parts of the laryngeal motor nucleus receive inputs from pontine nuclei, exactly that part of the nucleus ambiguus which is formed by motoneuron aggregation of the cricothyroid muscle (Schweizer et al. 1981). Only in this region could changes in the neuronal discharge activity be correlated with changes in the frequency of the echolocation calls in bats which compensated for Doppler-shifts (Rübsamen and Betz 1986). The pontine nuclei receive massive auditory input from the inferior colliculus of the midbrain (for rhinolophids: Schweizer 1981; for cats: Kawamura 1975) and from the auditory cortex (for cats: Diamond et al. 1969).

There are therefore two possible ways by which the auditory system can influence the motor control of vocalization via the pons nuclei: (1) The cerebellum receives an auditory input which is mediated by the lateral nuclei of the pons. Efferents from the cerebellum run to the frontal brain and 
thus may modify the extrapyramidal or the pyramidal motor systems, which send inputs to the nucleus ambiguus. (2) The nuclei of the pons send direct efferents to the nucleus ambiguus. The latter would enable a fast auditory mediated modification of the motor control of the frequency of echolocation pulses.

\section{Connections with respiratory centers}

The connections of the nucleus ambiguus with nuclei known to be involved in the generation of the respiration rhythm are predominantly reciprocally organized. Retrograde and anterograde labelling is found in the parabrachial nucleus and in the reticular formation. Our results correspond with data from neuroanatomical (Kalia 1981) and neurophysiological experiments (von Euler 1983).

\section{Reticular connections}

In all experiments, lightly labelled cells were distributed throughout the reticular formation of the medulla oblongata. This illustrates afferents of the reticular formation to the nucleus ambiguus. Detailed Golgi-studies in cats have shown the wide ranging organization of neuronal nets in the reticular formation and the interconnection of many brain stem nuclei by single neurons (Scheibel and Scheibel 1958; Valdere 1961).

\section{Conclusions}

Our results demonstrate a complex connectivity pattern of the nucleus ambiguus and the surrounding reticular formation with various brain structures. Motor centers, such as the motor cortex and the red nucleus may directly, or via interneurons in the reticular formation, influence laryngeal motoneurons. The motor pattern for vocalization, possibly generated in the pyramidal and extrapyramidal systems, may be modified by neurons of the lateral reticular formation of the medulla oblongata. This region also serves as a link to the cranial motor systems involved in the control of ear and nose movements which accompany the vocalization of echolocation pulses. The incorporation into this motor control system, of the periaqueductal gray and the cuneiform nucleus (obligatory relay stations in the vocalization system) is still a matter of conjecture. The auditory system may mediate information to the nucleus ambiguus from the inferior colliculus via the pontine nuclei and via the superior colliculus. The coordination of the vocalization and respiration systems is achieved by reciprocal connections with the parabrachial region and the reticular formation.
Acknowledgements. This investigation was supported by the Deutsche Forschungsgemeinschaft, SFB 204 (Gehör). We thank Ms C. Rühle for valuable assistance during the histological analyses and Ms F. Althaus, Ms H. Tscharntke and Ms B. Vellen for their persevering help in the documentation. We are grateful to Messrs G. Pollak and G. Neuweiler for critical comments on the manuscript.

\section{References}

Abols IA, Basbaum AI (1981) Afferent connections of the rostral medulla of the cat. A neural substrate for midbrain medullary interactions of the modulation of pain. J Comp Neurol 201:285-297

Adams JC (1977) Technical consideration on the use of the horseradish peroxidase as a neuronal marker. Neuroscience 2:141-145

Aitkin LM, Boyd J (1975) Response of single units in the cerebellar vermis of the cat to monaural and binaural stimuli. J Neurophysiol 38:418-429

Barillot C, Bianchi AL, Gogan P (1984) Laryngeal respiratory motoneurons: morphology and electrophysiological evidence of separate sites for exitatory and inhibitory synaptic inputs. Neurosci Lett 47:107-112

Bowden REM, Scheuer JL (1961) Comparative studies of the nerve supply of the larynx in eutherian mammals. Proc Zool Soc Lond 136:325-330

Boyd J, Aitkin LM (1976) Responses of single units in the pontine nuclei of cat to acoustic stimulation. Neurosei Lett 3:259-263

Chung JM, Keretter GA, Yezierski RP, Haber LH, Martin RF, Willis WD (1983) Midbrain nuclei projecting to the medial medulla oblongata in the monkey. J Comp Neurol 214:93-102

Diamond IT, Jones EG, Powell TPS (1969) Projections from the auditory cortex upon the diencephalon and brain stem in the cat. Brain Res 15:305-340

Edwards SB (1975) Autoradiographic studies of the midbrain reticular formation: Descending connection of nucleus cuneiformis. J Comp Neurol 161:341-359

Euler $C$ von (1983) On the functional organization of the generators of rhythmic motor synergy in breathing. In: Schläfke ME, Koepchen HP, See WR (eds) Central neuron environment. Springer, Berlin Heidelberg New York, pp 157-163

Henkel CK (1981) Afferent sources of a lateral midbrain tegmental zone associated with the pinnae in the cat as mapped by retrograde transport of HRP. J Comp Neurol 203:213-226

Henkel CK, Edwards SB (1978) The superior colliculus control of pinnae movement in the cat: Possible anatomical connections. J Comp Neurol 182:763-776

Henson OW (1970) The central nervous system. In: Wimsatt WA (ed) Biology of bats. Academic Press, New York London

Irvine DRF (1980) Acoustic input to neurons in feline red nucleus. Brain Res 200:165-169

Jürgens U, Ploog D (1970) Cerebral representation of vocalization in the squirrel monkey. Exp Brain Res 10:532-554

Jürgens U, Pratt R (1979) Role of periaqueductal gray in vocal expression of emotion. Brain Res 167:367-379

Kalia MP (1981) Anatomical organization of central respiratory neurons. Annu Rev Physiol 43:105-120

Kawamura K (1975) The pontine projections from the inferior colliculus in the cat. An experimental study. Brain Res 95:309-322 
Kuypers HGJM (1958) Some projections from the pericentral cortex to the pons and lower brain stem in monkey and chimpanzee. J Comp Neurol 110:221-251

Kuypers HGJM (1960) Central cortical projections to motor and somato-sensory cell groups. Brain $83: 161-184$

Lende RA (1969) A comparative approach to the neocortex: Localization in monotremes, marsupials and insectivores. Ann NY Acad Sci 167:262-276

Manthy PW (1982) Ascending input to the midbrain periaqueductal gray of primate. J Comp Neurol 211:50-64

Manthy PW (1983) Connection of midbrain periaqueductal gray in the monkey: I. Ascending efferent connections. J Neurophysiol 49:567-581

Mesulam M-M, Rosene DL (1979) Sensitivity in peroxidase neurohistochemistry: A comparative and quantitative study of nine methods. J Histochem Cytochem 27:763-773

Middelbrooks JC, Knudsen EI (1984) A neural code for auditory space in the cat's superior colliculus. J Neurosci $4: 2621-2634$

Pasaro R, Lobera B, Gonzales-Baron S, Delgado-Garcia JM (1983) Cytoarchitectonic organization of laryngeal motoneurons within the nucleus ambiguus of the cat. Exp Neurol $82: 623-634$

Rose M (1912) Histologische Lokalisation der Großhirnrinde der kleinen Säugetiere (Rodentia, Insektivora, Chiroptera). J Psychol Neurol 19:389 479

Rosene DL, Mesulam M-M (1978) Fixation variables in horseradish peroxidase neurohistochemistry. I. The effect of fixation time and perfusion procedures upon enzyme activity. J Histochem Cytochem 26:28-39

Rübsamen R, Betz M (1986) Control of echolocation pulses by neurons of the nucleus ambiguus of the rufous horseshoe bat, Rhinolophus rouxi. I. Single unit recordings in the ventral motor nucleus of the laryngeal nerves in spontaneously vocalizing bats. J Comp Physiol A 159:675-687

Rübsamen R., Schuller G (1981) Laryngeal nerve activity during pulse emission in the CF-FM-bat, Rhinolophus ferrumequinum. II. The recurrent laryngeal nerve. J Comp Physiol $143: 323-327$

Saper CB, Loewy AD (1980) Efferent connections of the parabrachial nucleus in the rat. Brain Res 197:291-317

Sawchenko PE (1983) Central connections of the sensory and motor nuclei of the vagus nerve. J Autonomic Nerv System $9: 13-27$
Scheibel ME, Scheibel AB (1958) Structural substrates for integrative patterns in the brain stem reticular core. In: Jasper $\mathrm{HH}$ (ed) The reticular formation of the brain. Little, Brown and $\mathrm{Co}$, Boston Massachusetts, pp 31-55

Schuller G (1977) Echo delay and overlap with emitted orientation sounds and Doppler-shift compensation in the bat, Rhinolophus ferrumequinum. J Comp Physiol 114:103-114

Schuller G (1979) Vocalization influences auditory processing in collicular neurons of the CF-FM-bat, Rhinolophus ferrumequinum. J Comp Physiol 132:39-46

Schuller G, Rübsamen R (1981) Laryngeal nerve activity during pulse emission in the CF-FM-bat, Rhinolophus ferrumequinum. I. Superior laryngeal nerve (external motor branch). J Comp Physiol 143:317-321

Schuller G, Suga N (1976) Laryngeal mechanisms for the emission of CF-FM-sounds in the Doppler-shift compensating bat, Rhinolophus ferrumequinum. J Comp Physiol 107:253-262

Schweizer H (1981) The connection of the inferior colliculus and the organization of the brain stem auditory system in the greater horseshoe bat (Rhinolophus ferrumequinum). J Comp Neurol 201:25-49

Schweizer H, Rübsamen R, Rühle C (1981) Localization of brain stem motoneurons innervating the laryngeal muscles in the rufous horseshoe bat, Rhinolophus rouxi. Brain Res $230: 41-50$

Shimozawa T, Sum X, Jen H-S (1984) Auditory space representation of the superior colliculus of the big brown bat, Eptesicus fuscus. Brain Res 311:289-296

Suga N, Schlegel P, Schimozawa T, Simmons J (1973) Orientation sounds evoked from echolocating bats by electrical stimulation of the brain. J Acoust Soc Am 54:793-797

Valdere F (1961) Reticular formation of the pons and medulla oblongata. A Golgi study. J Comp Neurol 116:71-100

Valdere F (1962) Reticular formation of the albino rat's brain stem, cytoarchitecture and corticofugal connections. $J$ Comp Neurol 119:25-53

Walberg F (1957) Do motor nuclei of the cranial nerves receive corticofugal fibers? An experimental study. Brain 80:597-605

Yoshida Y, Miyazaki T, Hirano M, Shin T, Tanaseki T (1982) Arrangement of motoneurons innervating the intrinsic laryngeal muscles of cats as demonstrated by HRP. Acta Otolaryngol 94:329-334 